\title{
A glutaminase isoform switch drives therapeutic resistance and disease progression of prostate cancer
}

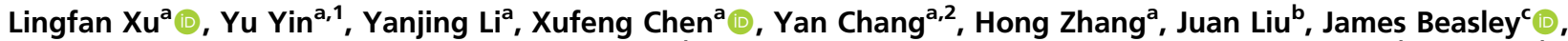

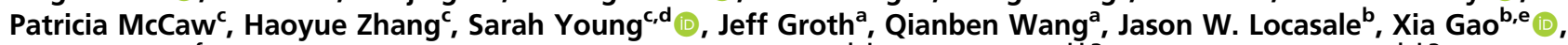

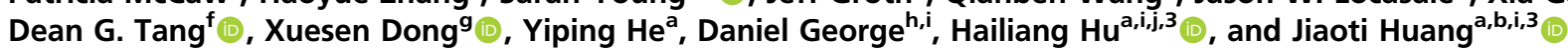

\begin{abstract}
${ }^{a}$ Department of Pathology, Duke University School of Medicine, Durham, NC 27710; bepartment of Pharmacology and Cancer Biology, Duke University School of Medicine, Durham, NC 27710; 'Duke University Health System Biochemical Genetics Laboratory, Durham, NC 27713; ${ }^{d}$ Department of Pediatrics,

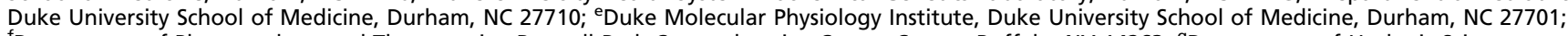
fDepartment of Pharmacology and Therapeutics, Roswell Park Comprehensive Cancer Center, Buffalo, NY 14263; 9 Department of Urologic Science, Vancouver Prostate Centre, University of British Columbia, Vancouver, BC, Canada BC V6H 3Z6; ${ }^{\text {h}}$ Department of Medicine, Duke University School of Medicine, Durham, NC 27710; 'Duke Cancer Institute, Duke University School of Medicine, Durham, NC 27710; and 'School of Medicine, Southern University of Science and Technology, Shenzhen, China 510515
\end{abstract}

Edited by Karen H. Vousden, Francis Crick Institute, London, United Kingdom, and approved February 24, 2021 (received for review June 19, 2020)

Cellular metabolism in cancer is significantly altered to support the uncontrolled tumor growth. How metabolic alterations contribute to hormonal therapy resistance and disease progression in prostate cancer $(\mathrm{PCa})$ remains poorly understood. Here we report a glutaminase isoform switch mechanism that mediates the initial therapeutic effect but eventual failure of hormonal therapy of $\mathrm{PCa}$. Androgen deprivation therapy inhibits the expression of kidney-type glutaminase (KGA), a splicing isoform of glutaminase 1 (GLS1) up-regulated by androgen receptor (AR), to achieve therapeutic effect by suppressing glutaminolysis. Eventually the tumor cells switch to the expression of glutaminase C (GAC), an androgenindependent GLS1 isoform with more potent enzymatic activity, under the androgen-deprived condition. This switch leads to increased glutamine utilization, hyperproliferation, and aggressive behavior of tumor cells. Pharmacological inhibition or RNA interference of GAC shows better treatment effect for castration-resistant PCa than for hormone-sensitive PCa in vitro and in vivo. In summary, we have identified a metabolic function of AR action in PCa and discovered that the GLS1 isoform switch is one of the key mechanisms in therapeutic resistance and disease progression.

prostate cancer | therapeutic resistance | glutaminase | GAC

A ndrogen receptor (AR) is required for the survival of prostate cancer (PCa) cells. Hormonal therapy, by suppressing androgen production and/or inhibiting AR function, is effective as a systemic treatment for PCa. Unfortunately, the therapy will eventually fail, and the development of castration-resistant PCa (CRPC) is inevitable (1-5). Newer drugs abiraterone and enzalutamide provide survival benefits for some CRPC patients, but treatment resistance and disease progression still occur. While most CRPCs still maintain adenocarcinoma histology (CRPC-adeno), some patients will develop an extremely aggressive PCa variant known as small-cell neuroendocrine carcinoma (SCNC) $(4,6)$.

$\mathrm{AR}$ is a physiological molecule and a key transcription factor in multiple organs which binds to numerous genes and activates many different downstream signaling pathways $(4,7,8)$. However, which of the AR functions is required for PCa cell survival and the underlying molecular mechanisms of tumor cell inhibition after AR blockade remain unclear. We hypothesize that among many different functions of AR activation, only some are directly related to PCa cell survival. If we can identify the downstream effectors of $\mathrm{AR}$ responsible for $\mathrm{PCa}$ survival, future treatments targeting only the downstream effectors instead of AR can be more specific and effective as well as independent of tumor cells' AR status, resulting in better efficacy and fewer side effects.

Although genomic, epigenetic, transcriptional, translational, and posttranslational alterations all contribute to cancer initiation and progression, the final common pathway that regulates tumor cell proliferation is cellular metabolism. Abnormal metabolism is a key cellular process that underlines cancer cells' malignant behavior (9-11). We hypothesize that AR regulates important metabolic pathways in PCa cells to support cell proliferation, and inhibition of AR by hormonal therapy disrupts the metabolic program of tumor cells, leading to tumor inhibition. We further hypothesize that tumor cells adapt to the androgen-deprived environment by restoring the metabolic function, resulting in tumor recurrence and disease progression. In this study, we show that AR up-regulates the expression of glutaminase 1 (GLS1), thus promoting glutamine utilization which is required for optimal growth of PCa cells. Hormonal therapy inhibits AR function, resulting in down-regulation of GLS1 which in turn suppresses glutamine utilization and tumor cell proliferation. Eventually the tumor cells

\section{Significance}

We report that androgen receptor (AR) promotes glutaminase 1 (GLS1) expression and glutamine utilization to support the survival of prostate cancer (PCa) cells. Hormonal therapy inhibits AR and decreases GLS1 expression and glutamine utilization to achieve therapeutic effect. Our results suggest that eventually the tumor cells switch GLS1 expression from the ARdependent KGA isoform to the androgen-independent and enzymatically more potent GAC isoform, which increases glutamine utilization and can contribute to the development of castration-resistant PCa. Our work has discovered a previously unknown AR function, a metabolic mechanism of hormonal therapy and an important therapeutic target more specific than AR. Targeting GLS1 may achieve similar therapeutic efficacy but without the side effects resulting from inhibiting AR's other important physiologic functions.

Author contributions: L.X., X.D., H.H., and J.H. designed research; L.X., Y.Y., Y.L., X.C., Y.C., Hong Zhang, J.L., J.B., P.M., Haoyue Zhang, S.Y., J.G., J.W.L., X.G., D.G.T., H.H., and J.H. performed research; L.X. contributed new reagents/analytic tools; L.X., Y.L., X.C., Q.W., X.D., Y.H., D.G., H.H., and J.H. analyzed data; and L.X., H.H., and J.H. wrote the paper.

Competing interest statement: J.H. is a consultant for or owns shares in the following companies: Kingmed, MoreHealth, OptraScan, Genetron, Omnitura, Vetonco, York Biotechnology, Genecode, and Sisu Pharma.

This article is a PNAS Direct Submission.

Published under the PNAS license.

${ }^{1}$ Present address: Department of Pathology, Anhui Medical University, Hefei, China 230001

${ }^{2}$ Present address: Institute of Clinical Pharmacology, Anhui Medical University, Hefei, China 230001.

${ }^{3}$ To whom correspondence may be addressed. Email: huhl@sustech.edu.cn or jiaoti. huang@duke.edu.

This article contains supporting information online at https://www.pnas.org/lookup/suppl/ doi:10.1073/pnas.2012748118/-/DCSupplemental.

Published March 22, 2021. 
express an androgen-independent GLS1 isoform resulting in tumor recurrence and disease progression.

\section{Results}

Androgen Deprivation Therapy Suppresses Proliferation and Metabolic Activities of PCa Cells while Inducing Glutamine Accumulation. Since $\mathrm{AR}$ is critical for PCa survival, we hypothesized that AR function likely supports certain metabolic activities required by the tumor cells. We took an unbiased approach to test this hypothesis by conducting a metabolite-profiling study of two hormone-sensitive PCa cell lines, LNCaP and LAPC4, cultured either with (normal fetal bovine serum, FBS) or without androgen (charcoal-stripped FBS which mimics the clinically used androgen deprivation therapy or ADT), respectively. Androgen withdrawal inhibited the proliferation of the tumor cells (SI Appendix, Fig. S1A) and resulted in remarkable changes in the levels of many metabolites and metabolic pathways in both cell lines at different time points, as illustrated by principal component analysis, heat map alignment, and pathway enrichment analysis (SI Appendix, Fig. S1 $B-F)$. They included metabolites involved in glycolysis, tricarboxylic acid (TCA) cycle, nonessential amino acids (NEAA), nucleotides, and pentose phosphate pathway (PPP).

The decrease in metabolite levels after androgen withdrawal was consistent with the observation that tumor cells' proliferation was significantly inhibited by ADT. Surprisingly, we observed a significant increase in glutamine levels in the two cell lines after ADT (Fig. $1 A$ and SI Appendix, Fig. S2 $A-D$ ). The increased intracellular glutamine could be caused by either enhanced glutamine synthesis or decreased glutamine catabolism by the tumor cells (SI Appendix, Fig. S2E). By examining the expression of glutamine transporter ASCT2 (encoded by $S L C 1 A 5$ gene) in $\mathrm{LNCaP}$ cells cultured with or without androgen, we found that

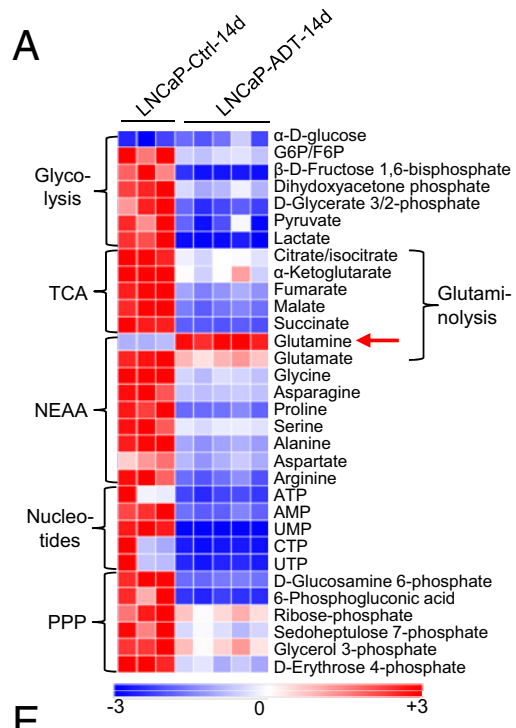

$E$
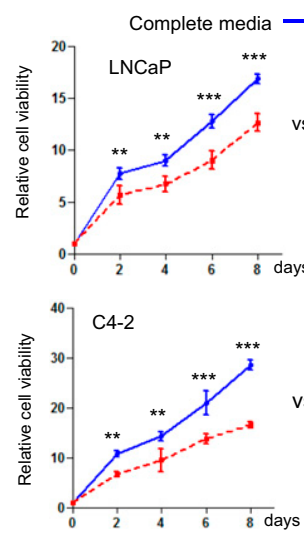

B

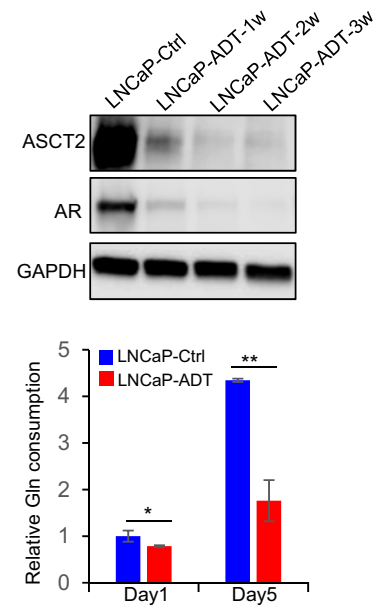

$\mathrm{F}$

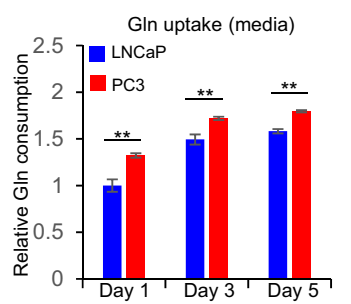

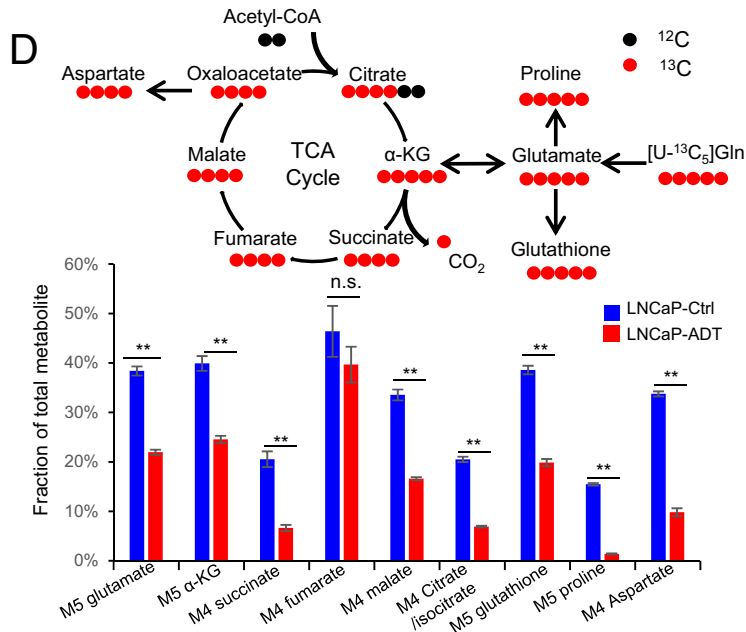

G

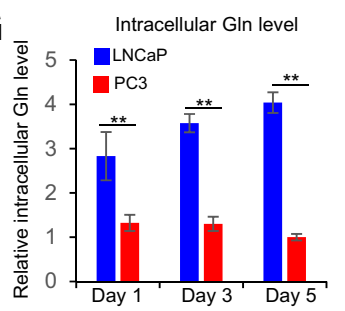

$\mathrm{H}$

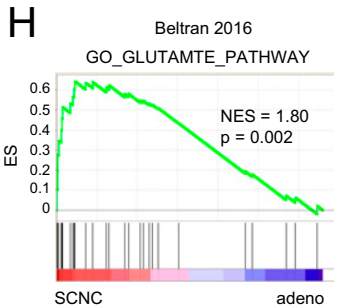

$J$

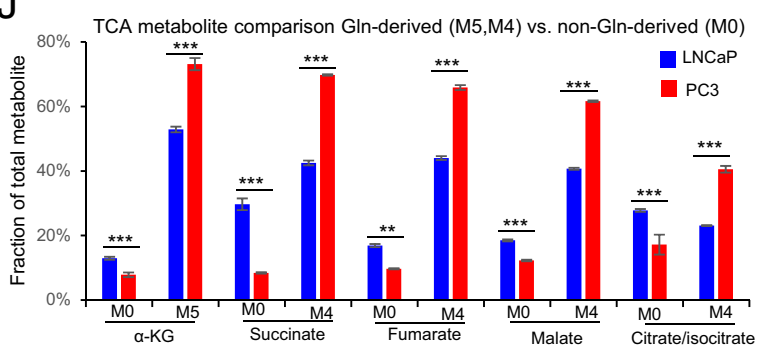

| GSE32967

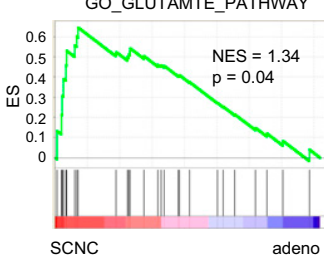

Fig. 1. Androgen deprivation inhibits glutamine catabolism, and therapy-resistant PCa cells are more addicted to glutamine. ( $A$ ) Heat map shows that ADT decreases levels of metabolites involved in important metabolism pathways ( $n=3$ and 5 replicates for the control and ADT groups, respectively). ( $B$ ) Western blot shows that glutamine transporter ASCT2, similar to AR, is decreased after ADT. (C) UPLC-MS analysis of cell culture medium to compare glutamine consumption with or without ADT ( $n=3$ cultures per group). (D) Tracing of ${ }^{13} \mathrm{C}$-labeled glutamine influx and mass isotopomer analysis of [U- $\left.{ }^{13} C_{5}\right]$ glutaminederived metabolites in LNCaP cells with or without ADT. $\alpha-K G, \alpha-k$ etoglutarate ( $n=3$ cultures per group). (E) Relative cell viability of LNCaP, PC3, C4-2, and C42MDVR cells cultured with or without glutamine ( $n=3$ replicates for two independent experiments). ( $F$ and $G)$ Ultra performance liquid chromatographymass spectrometry (UPLC-MS) analysis of glutamine uptake and intracellular glutamine levels in LNCaP and PC3 cells. Metabolites were extracted from culture medium and cell pellets at the indicated time points ( $n=3$ cultures per group). ( $H$ and $l)$ GSEA of "GO_GLUTAMATE_PATHWAY" gene sets to compare SCNC and adenocarcinoma in Beltran 2016 (12) and GSE32967. ES, enrichment score; NES, normalized enrichment score. ( $/$ ) Mass isotopomer analysis of TCA cycle metabolite abundance in LNCaP and PC3 cells ( $n=3$ cultures per group). M0 represents the non-Gln-derived (mainly glucose-derived) metabolite pool, whereas M5 and M4 represent the ${ }^{13} \mathrm{C}$-labled Gln-derived metabolite pool. Data are expressed as mean \pm SD. $* P<0.05, * * P<0.01$, and $* * * P<0.001$ by twotailed Student's $t$ test. n.s., not significant. 
ADT significantly decreased ASCT2 message RNA (mRNA) and protein expression (Fig. $1 B$ and SI Appendix, Fig. S2F) and inhibited glutamine uptake from the medium (Fig. $1 C$ ), suggesting that increased intracellular glutamine after ADT is not a result of increased glutamine uptake. This conclusion was further supported by the fact that varying extracellular glutamine concentration had little impact on ADT-induced growth arrest of tumor cells (SI Appendix, Fig. S2G).

Glutamine is an NEAA which can be synthesized from other nutrients by the enzyme glutamine synthetase (GS), in addition to direct uptake from the extracellular space. The ${ }^{13} \mathrm{C}_{5}$-labeled glutamine-tracing study showed a reduction of intracellular M5glutamine fraction after ADT, consistent with attenuated glutamine uptake. However, we noticed an increase in M0-glutamine fraction after ADT (SI Appendix, Fig. S2H), indicating increased glutamine synthesis inside the tumor cells. Indeed, we observed a stepwise elevation of GS expression after androgen withdrawal (SI Appendix, Fig. S2I). The ${ }^{13} \mathrm{C}_{6}$-labeled glucose-tracing analysis also showed that ADT resulted in increased glutamine production from glucose (SI Appendix, Fig. S2J). These findings suggest that in PCa cells, ADT inhibits glutamine uptake from the extracellular space but increases glutamine production intracellularly.

The intracellular level of glutamine can also be influenced by its catabolism. The ${ }^{13} \mathrm{C}_{5}$-labeled glutamine-tracing analysis revealed that ADT significantly reduced the levels of almost all the metabolites downstream of glutamine catabolism, including glutamate (M5), glutathione (M5), proline (M5), aspartate (M4), $\alpha$-ketoglutarate $(\alpha-\mathrm{KG})(\mathrm{M} 5)$, and other TCA cycle intermediates (M4) (Fig. 1D), suggesting that $\mathrm{ADT}$ suppresses glutamine utilization by the tumor cells. To determine if this may be responsible for the observed growth inhibition after ADT, we supplemented the culture media with the downstream metabolites NEAA (excluding glutamine) and $\alpha-\mathrm{KG}$ and observed a substantial rescue of ADT-induced growth inhibition (SI Appendix, Fig. S2 $K$ and $L$ ). Together, these studies demonstrate that a mechanism by which ADT inhibits tumor cell viability is in part through suppression of glutamine catabolism.

Advanced and Therapy-Resistant PCa Cells Are More Addicted to Glutamine. The above results suggest that AR promotes glutamine utilization which may be essential for PCa cell survival. Hormonal therapy, through inhibition of AR function, inhibits glutamine utilization, leading to inhibition of cellular proliferation. Since hormonally treated PCa always recurs as CRPC-adeno or $\operatorname{SCNC}(3,4)$, we hypothesized that the recurrent tumor cells may have regained the ability to utilize glutamine despite the absence of androgen. To test this hypothesis, we examined the degree of glutamine dependency across several PCa cell lines that represent different stages of PCa: LNCaP (AR-positive, androgen-sensitive) versus PC3 [AR-negative, castration-resistant, with features of SCNC $(13,14)]$ and C4-2 (castration-resistant but enzalutamidesensitive) versus C4-2MDVR (C4-2 cells that are enzalutamideresistant after long-term culture with the drug). Glutamine was required for optimal growth of all examined PCa cells (Fig. 1E). However, PC3 and C4-2MDVR cells, representing very advanced stages of the disease, showed higher degrees of dependency on glutamine than LNCaP and C4-2 cells (Fig. 1E). In contrast, glucose deprivation had much smaller effect on these PCa cells compared to glutamine deprivation (SI Appendix, Fig. S3A), suggesting that PCa cells become increasingly addicted to glutamine as the disease progresses. This conclusion was further supported by glutamine consumption study showing that PC3 and C42MDVR cells utilized more glutamine than $\mathrm{LNCaP}$ and C4-2 cells (Fig. $1 F$ and $G$ and SI Appendix, Fig. S3 $B$ and $C$ ), respectively. Glutamine addiction of advanced PCa was also observed in gene set enrichment analysis (GSEA), showing that the expression levels of genes involved in glutamate metabolism pathway were correlated with those genes associated with SCNC, the most advanced, therapy-resistant and lethal histologic variant of PCa (Fig. $1 H$ and $I$ ).

Our group has reported that in advanced $\mathrm{PCa}$, glucose is shunted toward the glycolytic pathway instead of entering the more energy-efficient TCA cycle $(15,16)$. However, more advanced PCa cells have higher levels of TCA cycle activity than hormonesensitive PCa (17), suggesting that they are able to efficiently use nutrient sources other than glucose to maintain the TCA cycle. We performed a ${ }^{13} \mathrm{C}$-labeled glutamine-tracing experiment which showed that the AR-negative PC3 cells and the enzalutamideresistant C4-2MDVR cells had more $\alpha-K G$ (M5) and other TCA cycle intermediates (M4) derived from glutamine, whereas LNCaP and C4-2 cells had more TCA cycle intermediates derived from nonglutamine sources (M0) (Fig. $1 J$ and SI Appendix, Fig. S3 D and $E$ ). These data suggest that instead of glucose, glutamine becomes the major carbon source for the maintenance of TCA cycle in advanced and therapy-resistant PCa (SI Appendix, Fig. S3F).

The critical role of glutamine in advanced PCa was also demonstrated by the metabolite-profiling study showing that glutamine and TCA cycle-related metabolic pathways were enriched in tumor cells that are AR-negative or show features of prostate SCNC such as PC3 and C4-2/N-Myc cells [C4-2 cells overexpressing $N$-Myc (18-20)] (SI Appendix, Fig. S4A). We also observed that glutamine deprivation led to distinct metabolic changes in AR-negative PC3 cells and AR-positive LNCaP cells (SI Appendix, Fig. S4 $B-F$ ), further supporting the notion that primary and advanced tumors respond differently to glutamine withdrawal.

Differential Expression of Glutaminase Isoforms in PCa of Different Stages. The results above suggest that hormonal therapy inhibits PCa cell survival in part through inhibition of glutamine utilization, but advanced and therapy-resistant $\mathrm{PCa}$ cells regain the ability to use glutamine which may contribute to therapy resistance and disease progression. To investigate the underlying mechanism, we examined the expression of glutaminase (GLS, including GLS1 and GLS2), the first rate-limiting enzyme in glutaminolysis (21-23), in a panel of PCa cell lines. GLS2 can be a tumor suppressor $(24,25)$, and our study showed that it was not expressed in PCa tissues or a panel of PCa cell lines examined (SI Appendix, Fig. S5 $A$ and $B$ ). The other glutaminase, GLS1, has two isoforms, kidney-type glutaminase (KGA) and glutaminase $\mathrm{C}$ (GAC), due to differential RNA splicing (26) (Fig. 2A). GAC is much more potent than KGA in catalyzing the conversion of glutamine to glutamate in vitro and in vivo (27). We found that KGA was highly expressed in AR-positive $\mathrm{PCa}$ cells, such as $\mathrm{LNCaP}$, VCaP, LAPC4, and C4-2, whereas GAC expression predominated in the more advanced and therapy-resistant C4-2MDVR and LNCaP95 cells (both resistant to enzalutamide) as well as $\mathrm{PCa}$ cells that are AR-negative and/or with neuroendocrine/SCNC features such as PC3, NCI-H660, and DU145. (Fig. $2 B$ and SI Appendix, Fig. S5 C and $D$ ). We also observed a correlation of the enzymatically strong GAC isoform with increasing Gleason scores in the TCGA database. The mRNA level of GAC, but not KGA, showed a stepwise increase with increasing Gleason scores (SI Appendix, Fig. S5E). Additionally, patients whose tumors harbored high levels of GAC had much shorter survival than those whose tumors had low GAC (Fig. 2C). In contrast, KGA levels did not show such clinical relevance (SI Appendix, Fig. S5F).

Adenocarcinoma of the prostate consists of bulk luminal-type tumor cells that are AR-positive and a minor population of ARnegative neuroendocrine (NE) tumor cells. Our group has recently demonstrated that $\mathrm{CXCR} 2$, an interleukin-8 receptor, is specifically expressed in NE tumor cells of human PCa (28). In the same study, we showed that NE tumor cells possess molecular features associated with aggressive cancer phenotypes such as stemness, epithelial-mesenchymal transition, and angiogenesis. We purified NE tumor cells from fresh primary human $\mathrm{PCa}$ tissue (prostatectomy specimens) by fluorescence-activated cell 
A

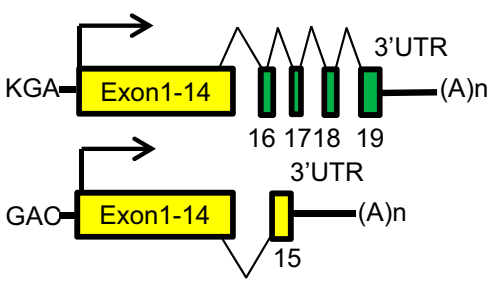

D

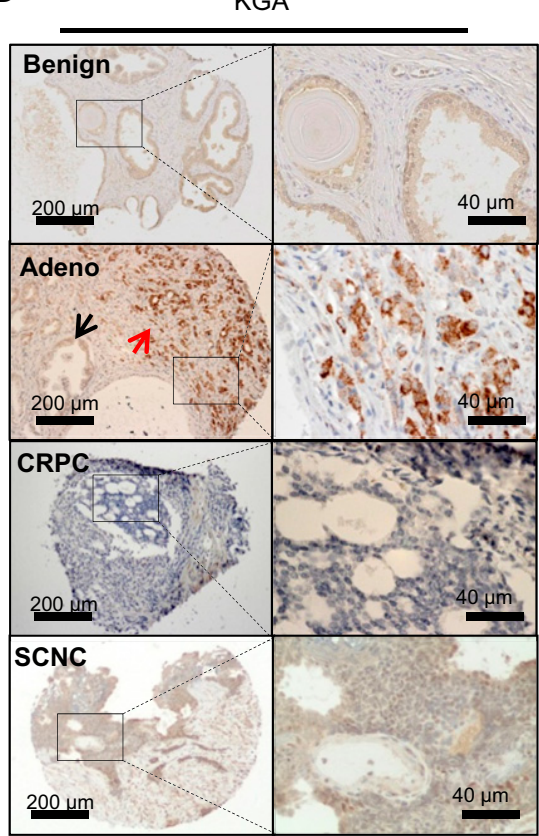

B

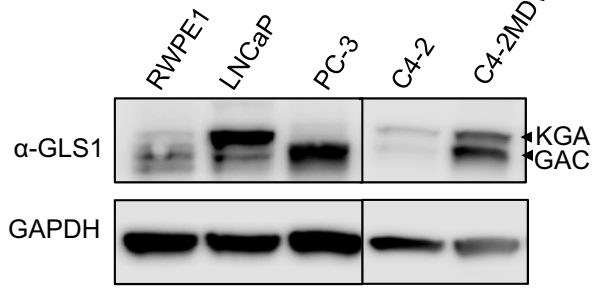

GAC

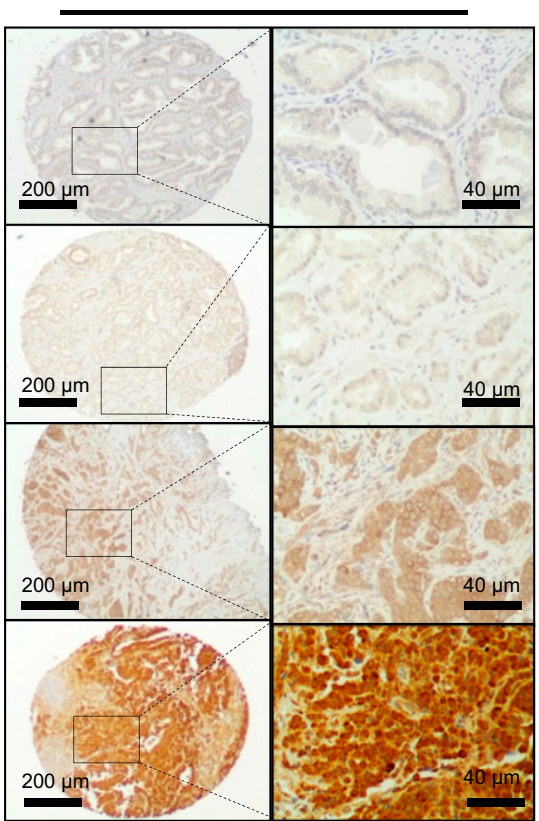

C

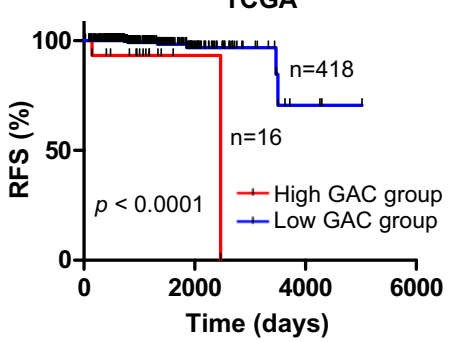

KGA

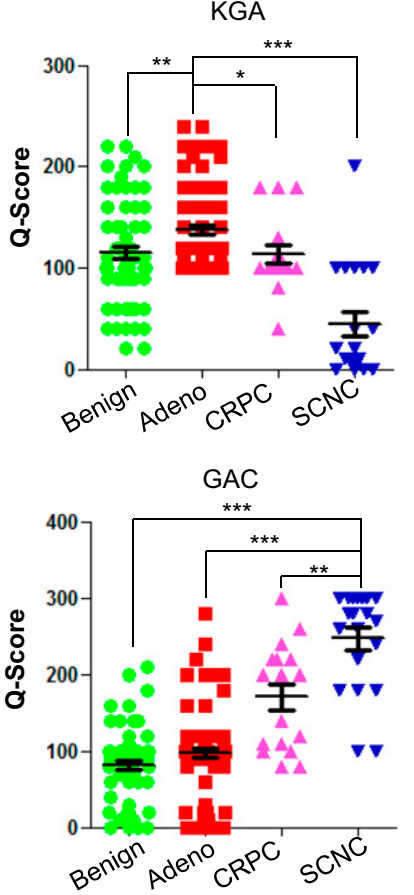

E

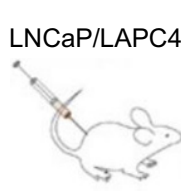

Treatment-
naïve tumo
Tumor recurs

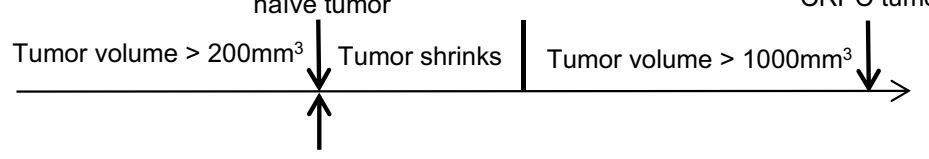

F

LNCaP xenografts

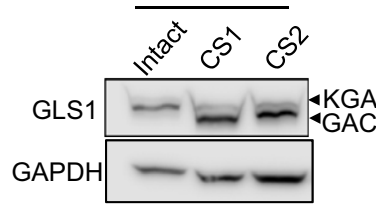

LAPC4 xenografts

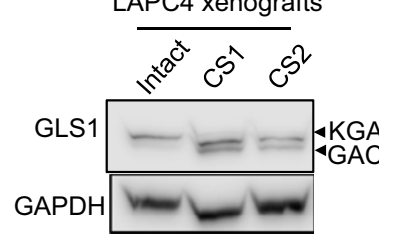

Fig. 2. Isoform switch of GLS1 during disease progression of PCa. (A) Gene structures of $K G A$ and GAC isoforms of GLS1. (B) Western blot to determine KGA and GAC protein levels in RWPE1, LNCaP, PC3, C4-2, and C4-2MDVR cells with an anti-GLS1 antibody that recognizes both isoforms. (C) Recurrence-free survival of patients stratified by GAC mRNA expression in the TCGA dataset. Log-rank (Mantel-Cox) test. (D) Representative images of KGA and GAC IHC staining of tissue microarrays containing benign prostate tissue (Benign, $n=67$ ), primary prostate adenocarcinoma (Adeno, $n=76$ ), castration-resistant PCa (CRPC, $n=17$ ), and small-cell neuroendocrine PCa (SCNC, $n=20$ ). Black and red arrows denote benign and tumor compartments (KGA/Adeno). The Right panels are scores for KGA and GAC staining (staining intensity $\times$ the percentage of positive cells) by using the Quick-Score (Q-Score) system. $P$ values were calculated using unpaired $t$ test. Scale bars are shown as indicated. (E) Hematoxylin and eosin (H\&E) staining and IHC analysis of KGA and GAC expression in mouse LNCaP xenograft tumors before castration (Intact) and in recurrent tumors after castration (CS) $(n=4)$. Upper panel shows the experimental procedure. (Scale bar, $40 \mu \mathrm{m}$.) Bottom Right panel shows a plot of Q-Score for KGA and GAC staining in each xenograft tumor group. (F) Western blot analysis of KGA and GAC protein levels in hormone-sensitive xenograft tumors (Intact) and castration-resistant xenograft tumor (CS) with GLS1 antibody. $P$ values were calculated using two-tailed Student's $t$ test. ${ }^{*} P<0.05, * * P<0.01$, and $* * * P<0.001$. 
sorting using an anti-CXCR2 antibody and determined the ratios of KGA and GAC mRNA levels using isotype-specific primers (29). Luminal-type tumor cells were purified and used as controls. In four of five samples, the ratio of GAC/KGA was dramatically increased in the CXCR2-positive NE cells compared to that in the CXCR2-negative luminal cells (SI Appendix, Fig. $\mathrm{S} 5 G$ ), further supporting the notion that GAC expression is associated with a more aggressive phenotype.

The above results were confirmed in human $\mathrm{PCa}$ tissue by immunohistochemical (IHC) staining of tissue microarrays (TMAs) with KGA- and GAC-specific antibodies, respectively. Consistent with the in vitro data, KGA expression was the highest in untreated (hormone-naive) prostate adenocarcinoma and decreased in the more advanced and aggressive CRPC and SCNC, whereas GAC expression was lower in hormone-naive adenocarcinomas and higher in CRPC and SCNC (Fig. 2D). Notably, KGA expression was inversely correlated with GAC expression in PCa across different clinical stages (hormone-sensitive adenocarcinoma, CRPC and SCNC) (SI Appendix, Fig. S5H). These results demonstrate that the two isoforms of GLS1 display differential expression patterns in different stages of $\mathrm{PCa}$. Specifically, the enzymatically weaker KGA isoform is expressed early in the disease process, and as the disease progresses to advanced and therapy-resistant stages, the enzymatically stronger GAC becomes the predominant isoform in tumor cells.

To determine whether the GLS1 isoform switch from KGA to GAC during disease progression can be observed in vivo longitudinally, we employed hormone-sensitive xenograft tumor models wherein established LNCaP and LAPC4 tumors in mice regressed upon castration but eventually recurred as CRPC (30) (SI Appendix, Fig. S5 I and J). IHC as well as Western blot analyses of the xenograft tumors showed that in comparison to tumors from intact mice (hormone-sensitive), the recurrent tumors after castration (CRPC) expressed lower levels of KGA but higher levels of GAC (Fig. $2 E$ and $F$ ), again demonstrating a GLS1 isoform switch during the process of therapy resistance and disease progression.

The Isoform Switch from KGA to GAC Drives Therapy Resistance. We next determined whether the isoform switch of GLS1 from KGA to GAC increases glutamine utilization in tumor cells and leads to castration resistance. We specifically knocked down KGA and GAC, respectively, using short-hairpin RNAs (shRNAs) in LNCaP and PC3 cells (Fig. $3 A$ and $B$ ). LNCaP cells were more sensitive to the reduction of KGA in terms of cell proliferation and glutamine utilization (Fig. $3 A$ and $C$ ), consistent with our observation that KGA is the dominant isoform of GLS1 in androgen-dependent cells, including LNCaP cells. In PC3 cells, in contrast, loss of GAC caused a significant decrease of glutamine utilization and a dramatic cell growth arrest, while knocking down KGA had little effect (Fig. $3 B$ and $D$ ). To further validate the function of GAC in tumor cells, we generated CRISPR/Cas9mediated GLS1-deficient cells and introduced back KGA and GAC, respectively (Fig. $3 E$ and $F$ and SI Appendix, Fig. S5K). PC3 cells exhibited a greater degree of dependency on GLS1 gene compared to LNCaP cells (Fig. $3 E$ and $F$ ). Reexpression of KGA partially rescued cell proliferation in both LNCaP and PC3 cells (Fig. $3 E$ and $F$ ). In contrast, cell proliferation was remarkably increased after reintroducing GAC in the two cell lines (Fig. $3 E$ and $F$ ). These results suggest that the stronger enzymatic activity of GAC confers the rapid proliferation of advanced PCa through enhanced glutamine catabolism. Additionally, in both cell models, the ratio of glutamate/glutamine (reflecting the kinetics of conversion from glutamine to glutamate) was higher in GACexpressing cell models (Fig. $3 G$ and $H$ ), supporting previous reports that GAC possesses a much stronger enzymatic activity than KGA.
To determine whether the switch from KGA to GAC drives castration resistance, we generated LNCaP cells stably overexpressing the GAC isoform and subjected the cells to androgen deprivation (Fig. 3I). We observed that GAC overexpression in LNCaP cells partially rescued the proliferation of $\mathrm{LNCaP}$ cells under androgen-deprived conditions (Fig. 3J). This observation was confirmed in vivo with the xenograft tumor model (Fig. 3I). Overexpression of GAC increased the sizes of LNCaP xenograft tumors in intact animals, and such tumors were more resistant to castration than control tumors (Fig. $3 K$ and $L$ ). Furthermore, increased proliferation of $\mathrm{LNCaP}$ cells overexpressing GAC was abrogated by depleting glutamine and enhanced by increasing glutamine concentration in the androgen-deprived medium (Fig. 3M). In contrast, changing glucose concentration had little effect under the same experimental conditions (Fig. $3 N$ ), suggesting that overexpression of GAC leads to increased utilization of glutamine and confers the tumor cells resistance to ADT.

GLS1 Inhibitor CB-839 Preferentially Suppresses Advanced and Therapy-Resistant PCa. Having documented that advanced and therapy-resistant PCa overexpress GAC and are more addicted to glutamine, we next studied if therapy-resistant $\mathrm{PCa}$ is more sensitive to GLS1 inhibition than primary PCa. We tested a specific GLS1 inhibitor CB-839 (i.e., Telaglenastat, Calithera Biosciences) $(31,32)$, which is in clinical trials for multiple solid tumors (\#NCT02071862), including renal cell carcinoma, for its potential in inhibiting $\mathrm{PCa}$. CB-839 had a more potent inhibitory effect on PC3 cells ( $\mathrm{IC}_{50}=481 \mathrm{nM}$ ) than on RWPE1 (a benign prostate epithelial cell line) and $\mathrm{LNCaP}$ cells $\left(\mathrm{IC}_{50}>1,000 \mathrm{nM}\right)$ (Fig. 4A). Furthermore, CB-839 more potently inhibited cell growth, colony formation, and invasion of advanced $\mathrm{PCa}$ cells than those of hormone-dependent cells (Fig. $4 B-D$ and SI Appendix, Fig. S6 $A$ and $B$ ), supporting our finding that advanced cancer cells are more addicted to glutamine and more sensitive to GLS1 inhibition. In vivo xenograft models further confirmed the more potent inhibitory effect of CB-839 on PC3 xenografts than LNCaP tumors (Fig. $4 E$ and $F$ ) as determined by tumor sizes and the proliferation marker Ki67 (SI Appendix, Fig. S6C). In addition, ${ }^{13} \mathrm{C}_{5}$-labeled glutamine-tracing experiments showed that CB-839 inhibited glutamine utilization in both LNCaP and PC3 cells, but the inhibitory effect was more pronounced in the AR-negative, androgen-independent PC3 cells (Fig. $4 G$ and $S I$ Appendix, Fig. S6 $D$ and $E$ ). These results suggest that inhibition of glutamine catabolism is more efficacious in advanced and hormonal therapy-resistant $\mathrm{PCa}$ than in primary $\mathrm{PCa}$.

Next, we determined if CB-839 has different specificity for the two isoforms of GLS1. We employed the previously generated cell lines specifically expressing KGA or GAC (Fig. $3 E$ and $F$ and SI Appendix, Fig. S5K) and subjected them to CB-839 treatment. CB-839 showed a greater inhibitory effect on GACexpressing cell lines (Fig. 4H).

AR and MYC Cooperatively Contribute to the GLS1 Isoform Switch. A unique feature of $\mathrm{PCa}$ is that the tumor cells require androgen for their survival $(17,33)$. Given that the expression of KGA is associated with a hormone-sensitive state while that of GAC is associated with castration resistance after AR-targeted therapy, we studied if AR regulates GLS1 alternative splicing during disease progression. We first analyzed the AR ChIP-sequencing datasets $(34,35)$ and found a strong AR-binding peak in the $3^{\prime}$ untranslated region (UTR) of GLS1 in dihydrotestosterone (DHT)-stimulated LNCaP cells (Fig. 5A). Detailed DNA sequence analysis revealed two putative androgen response elements (AREs) in this region (SI Appendix, Fig. S7A), and ChIP-qPCR verified the binding of AR to this region (Fig. $5 B$ ). We then cloned 3'UTR of GLS1 containing the two AREs into a cytomegalovirus (CMV)-driven Renilla luciferase reporter construct (pRL) and measured the luciferase activity in the presence or absence of 

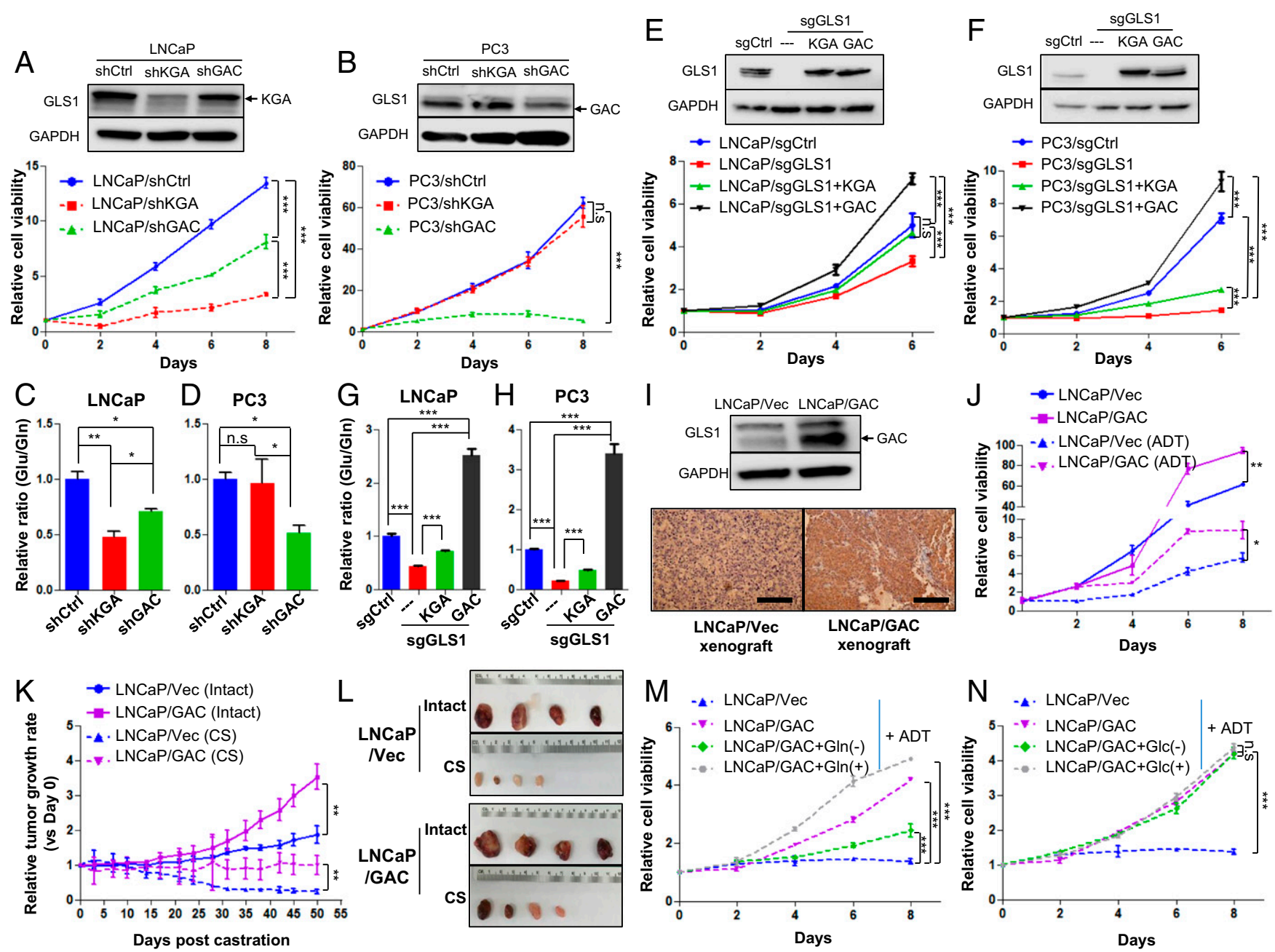

Fig. 3. The GLS1 isoform switch confers castration resistance to PCa cells. ( $A$ and $B$ ) Western blots show KGA and GAC expression of LNCaP and PC3 cells transduced with KGA-specific, GAC-specific, or control shRNAs and the corresponding cell viability for each cell line $(n=3$ replicates for two independent experiments). ( $C$ and $D$ ) Glutamine utilization indicated by the ratio of intracellular glutamate/glutamine of cells in $A$ and $B$ ( $n=3$ cultures per group). ( $E$ and F) Cell viability after knocking out GLS1 and introducing KGA and GAC back, respectively ( $n=3$ replicates for two independent experiments). ( $G$ and $H$ ) Enzymatic activity of KGA and GAC as measured by the ratio of intracellular glutamate/glutamine in cells described in $E$ and $F$ ( $n=3$ cultures per group). ( $I)$ Western blot and IHC staining of GAC overexpression in LNCaP cells and the corresponding xenograft tumor. $(J)$ Cell viability of LNCaP cells overexpressing GAC and LNCaP/Nec control in regular medium or charcoal-stripped medium (ADT) ( $n=3$ replicates for two independent experiments). ( $K$ and $L$ ) Xenograft tumor growth curves with or without surgical castration (CS) and representative images of tumor size ( $n=4$ in each group). (M and $N)$ Cell viability of LNCaP/ Vec and LNCaP/GAC treated with glutamine (GIn)/glucose (Glc) depletion or supplemented glutamine/glucose in the charcoal-stripped medium (ADT) $(n=3$ replicates for two independent experiments). Data are expressed as mean \pm SD. $* P<0.05, * * P<0.01$, and $* * * P<0.001$ by two-tailed Student's $t$ test. n.s., not significant.

DHT. DHT up-regulated luciferase activity of the construct containing the GLS1-wide-type-3'UTR in comparison to the vector control (SI Appendix, Fig. S7B). Three other constructs containing the GLS1-3'UTR with deletions of each ARE individually or in combination were generated, and luciferase reporter assays showed that each ARE mutation diminished the luciferase activity and the mutation combination had the strongest effect (SI Appendix, Fig. S7B), suggesting that AR binds to the AREs of GLS1 and up-regulates its expression. Additionally, we found that ADT of $\mathrm{LNCaP}$ cells repressed GLS1 expression (both KGA and GAC), and adding back DHT restored the protein levels of GLS1 (Fig. $5 C$ ), further supporting the conclusion that GLS1 is an ARregulated gene. These results were also consistent with our previous finding that $\mathrm{ADT}$ leads to glutamine accumulation by inhibiting glutamine catabolism (Fig. $1 A$ ).

To investigate if AR activity regulates the expression of the two isoforms differentially, we knocked down or overexpressed AR, respectively, in LNCaP cells. Although both KGA and GAC levels were affected, AR knockdown or overexpression decreased and increased the ratio of KGA/GAC (Fig. $5 D-F$ ), respectively. To further determine if AR could affect GLS1 mRNA splicing, we performed a minigene assay using a GLS1 minigene reporter plasmid covering exon 14 to exon 19 of GLS1 (SI Appendix, Fig. $\mathrm{S} 7 C$ ). The plasmid was cotransfected with either a small-interfering RNA targeting $A R$ (siAR) into AR-positive $\mathrm{LNCaP}$ cells or exogenous $A R$ into AR-negative PC3 cells. We showed that knocking down or overexpressing AR significantly up-regulated or reduced $K G A$ transcript levels but had little impact on $G A C$ transcript levels (Fig. 5G). These results suggest that GLS1 RNA splicing is coupled to GLS1 gene transcription governed by AR. Specifically, activated AR binds directly to $G L S 1$ to up-regulate its transcription and simultaneously regulate alternative splicing to favor the expression of the KGA isoform (SI Appendix, Fig. S7D).

The above results reveal AR's metabolic function in PCa and how hormonal therapy inhibits KGA expression and glutamine catabolism to achieve therapeutic effect. We then sought to 


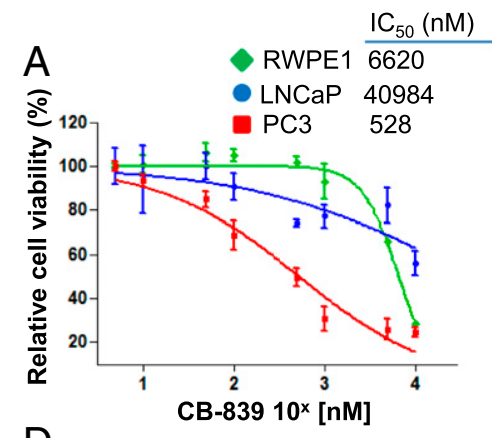

D BF CB-839
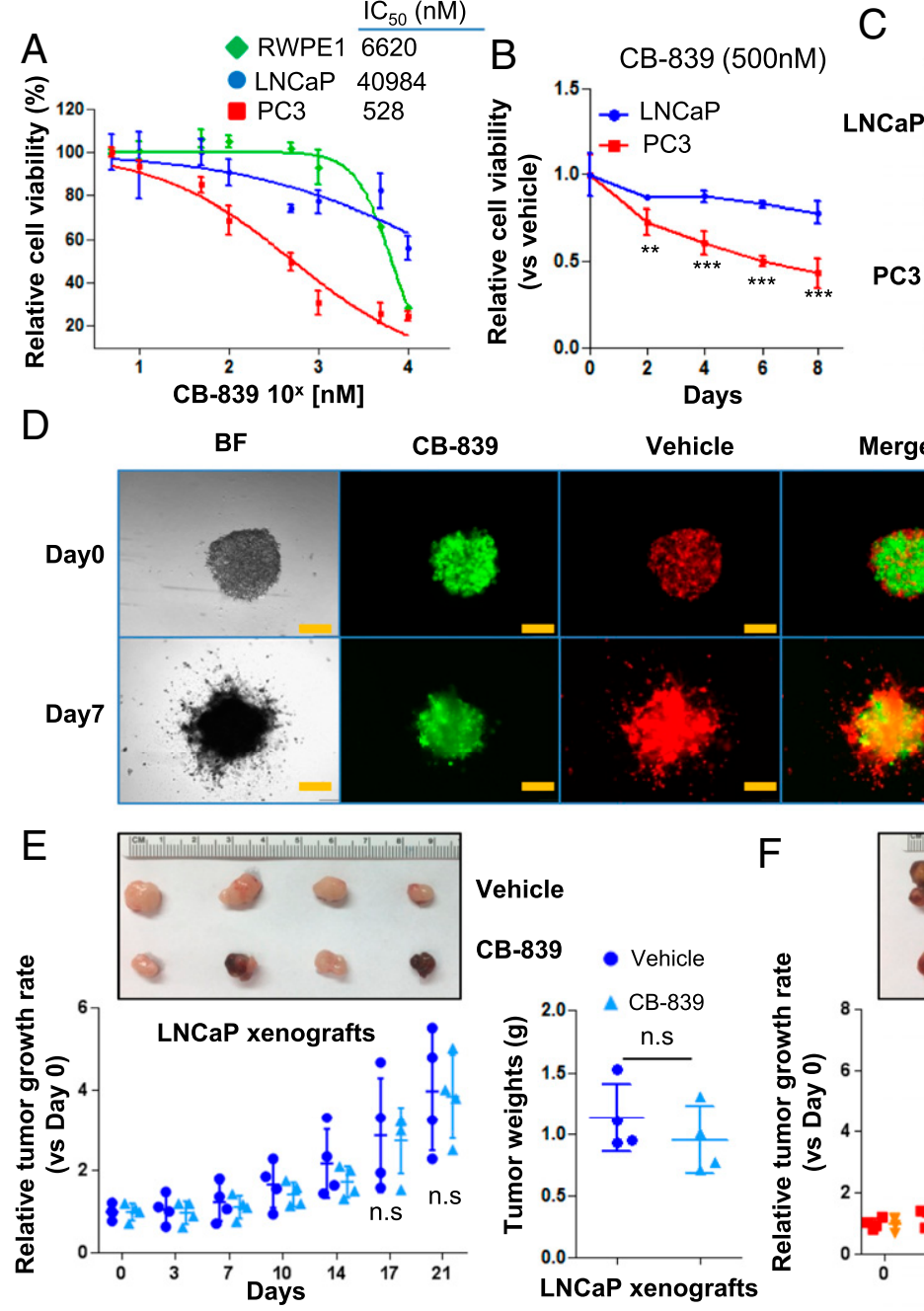

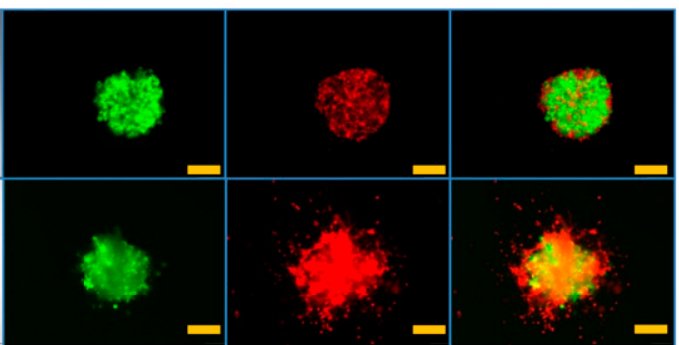

Vehicle
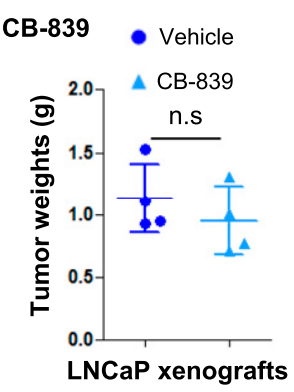

F
Vehicle CB-839

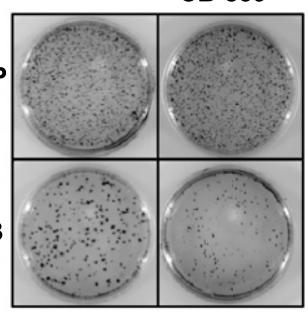

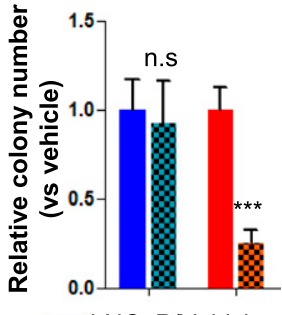

- LNCaP/Vehicle 00 LNCaP/CB-839 PC3/Vehicle 000 PC3/CB-839

G

Glutamine-derived (M4/M5) metabolites of glutaminolysis

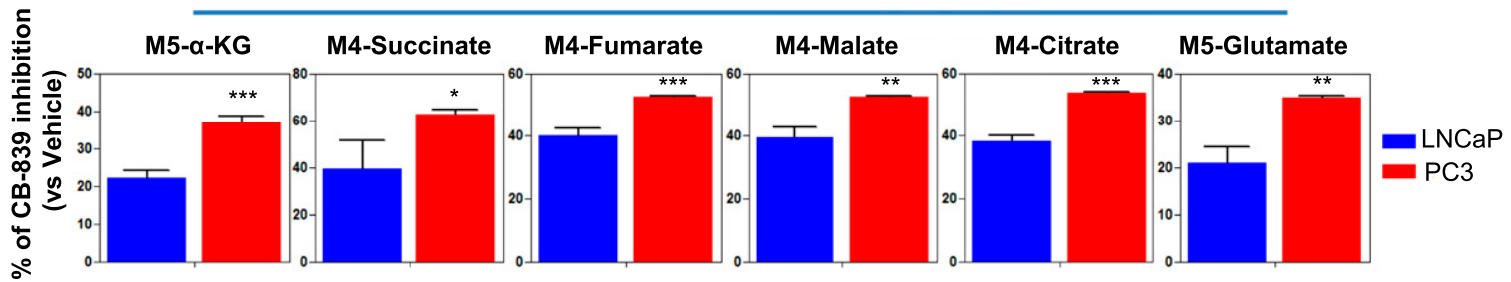

$\mathrm{H}$
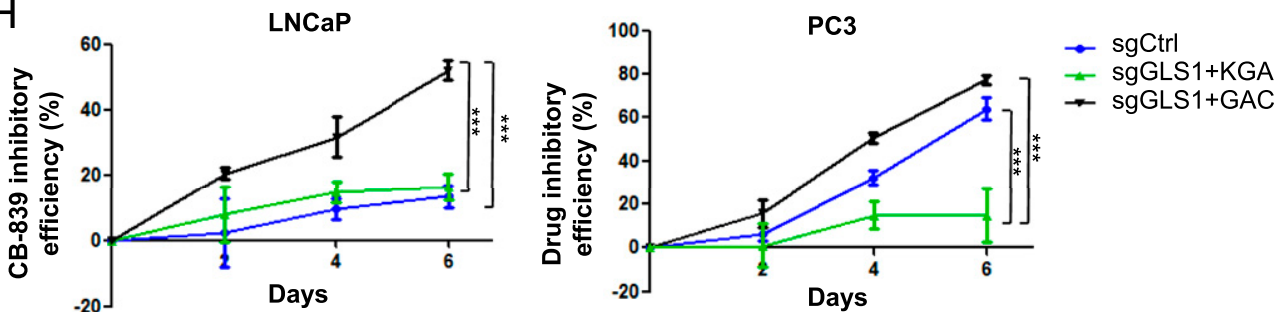

Fig. 4. GLS1 inhibitor CB-839 preferentially inhibits therapy-resistant PCa. (A) IC 50 of CB-839 in RWPE1, LNCaP, and PC3 cells after $72 \mathrm{~h}$ of drug treatment $(n=$ 3 replicates for two independent experiments). (B) Cell viability of LNCaP and PC3 cells treated with $500 \mathrm{nM}$ CB-839 ( $n=3$ replicates for two independent experiments). (C) Colony formation of LNCaP and PC3 cells treated with CB-839 (500 nM) for $14 \mathrm{~d}$ ( $n=3$ replicates for two independent experiments). (D) Spheroid invasion assay showing the inhibitory effect of CB-839 on the migration of PC3 cells ( $n=3$ replicates for two independent experiments). Representative images (Left) and quantifications of relative cell invasion (Right) are shown. Cells labeled with RFP or GFP denote those cultured with vehicle or CB839, respectively. (Scale bar, $200 \mu \mathrm{m}$.) (E and $F$ ) Representative images of tumor sizes (Top) and xenograft tumor growth curves (Bottom Left) and tumor weights (Bottom Right) with placebo or CB-839 oral administration ( $n=4$ in each group). (G) Mass isotopomer of ${ }^{13} \mathrm{C}$-glutanime-tracing analysis showing the inhibitory efficacy of CB-839 on glutaminolysis in LNCaP and PC3 cells $(n=3$ cultures per group). (H) CB-839 inhibitory efficacy in LNCaP and PC3 cells expressing KGA only or GAC only in comparison to control single guide RNA (sgCtrl) ( $n=3$ replicates for two independent experiments). Data are depicted as mean \pm SD. $* P<0.05, * * P<0.01$, and $* * * P<0.001$ by two-tailed Student's $t$ test. n.s., not significant. 

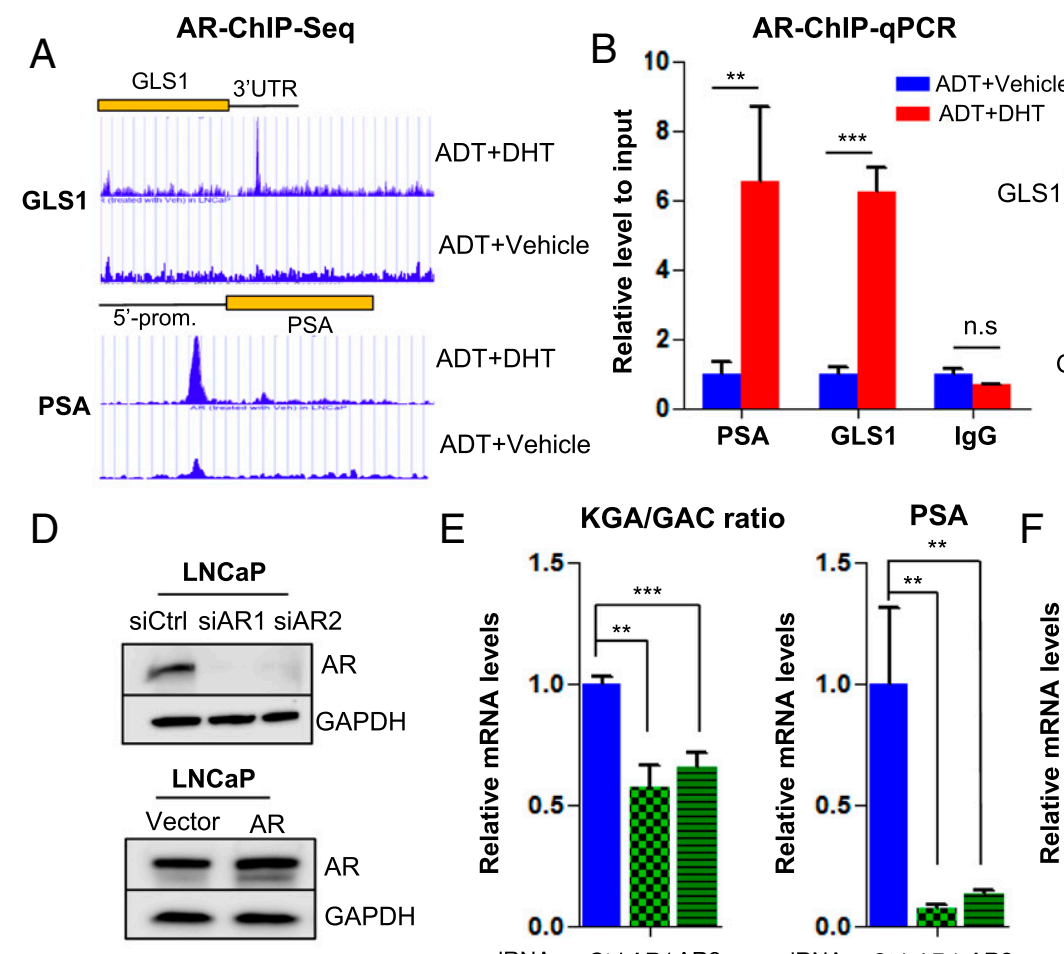

C
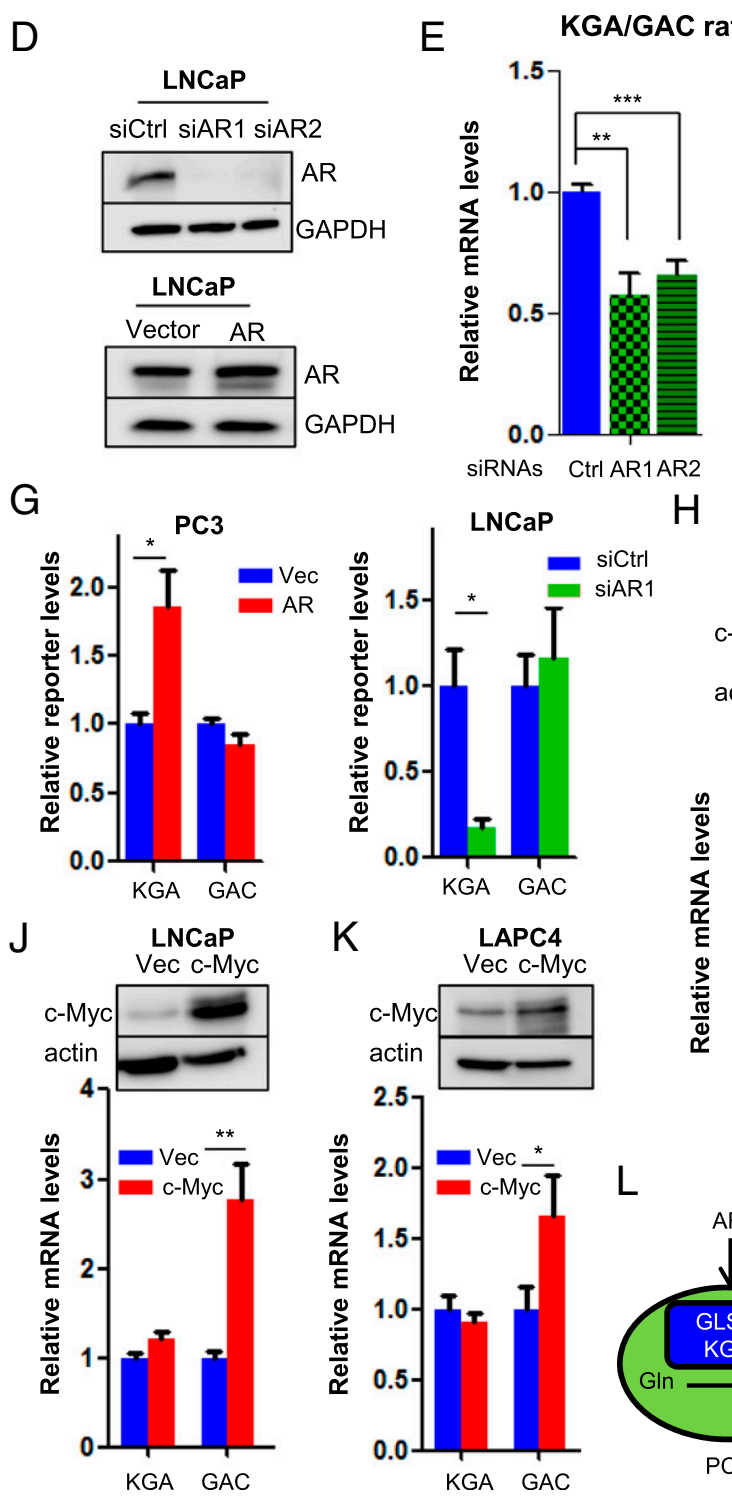

$\mathrm{H}$
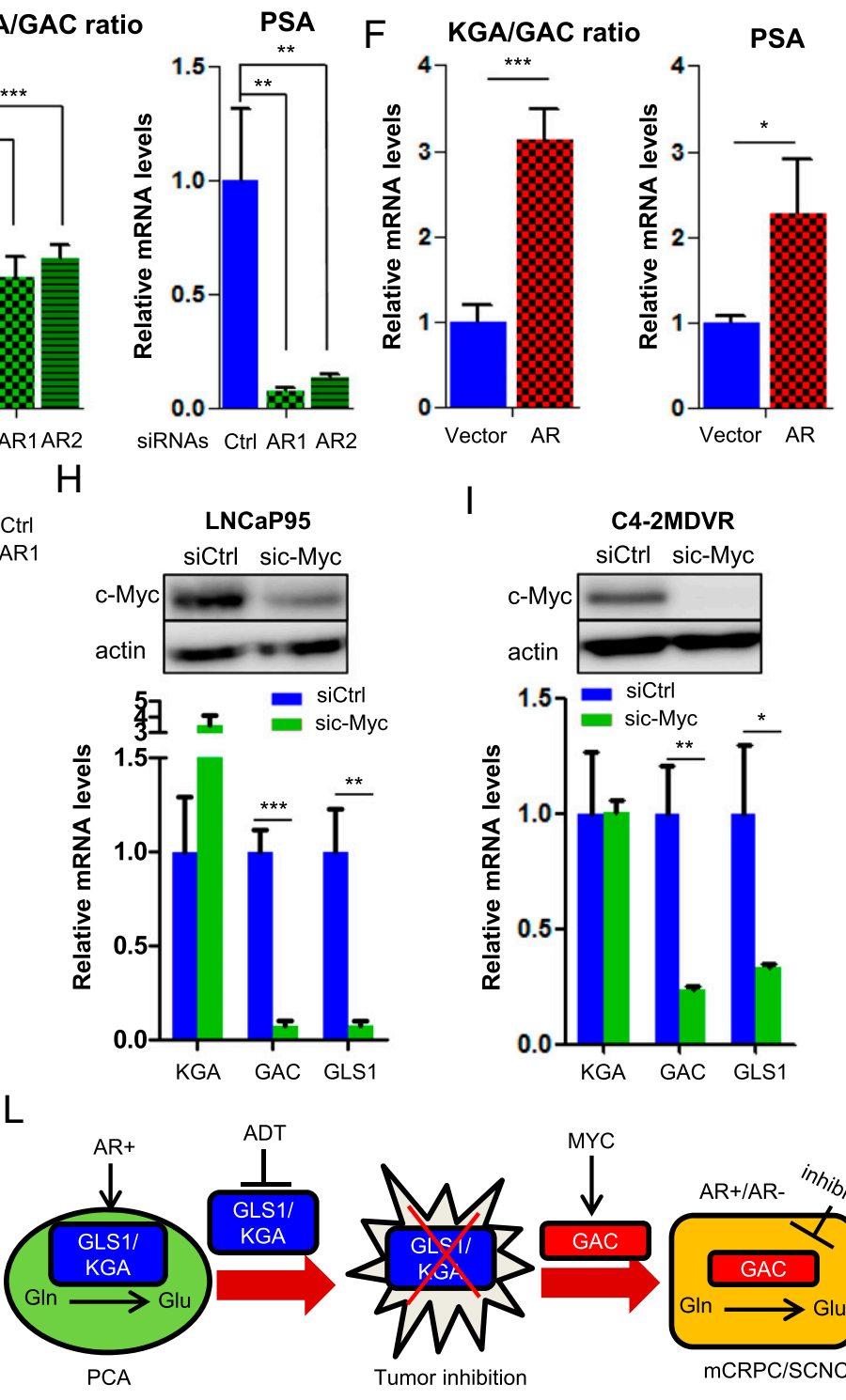

Fig. 5. AR and MYC cooperatively regulate GLS1 isoform switch. (A) Identification of AR-binding sites in GLS1-3'UTR by ChIP sequencing. Prostate-Specific Antigen (PSA) is used as a positive control. (B) ChIP-qPCR showing AR binding to GLS1-3'UTR upon DHT stimulation. (C) Western blot showing KGA and GAC expression in LNCaP cells treated with ADT and followed by $10 \mathrm{nM}$ DHT at the indicated time points. (D) Western blot showing AR expression in LNCaP cells transduced with siRNAs or complementary DNA (CDNA) plasmid expressing AR. ( $E$ and $F$ ) Transcript ratio of KGA/GAC in LNCaP cells after knockdown or overexpression of AR. (G) GLS1 minigene reporter was cotransfected with either AR CDNA plasmid in PC3 cells or AR-targeting siRNA in LNCaP cells. Transcript levels of isoforms were detected by qPCR by using $K G A$ - and GAC-specific primer pairs. ( $H$ and $I$ ) Western blot showing knockdown of c-Myc by specific siRNA pool in LNCaP95 and C4-2MDVR cells (Top) and transcript levels of KGA, GAC, and GLS1 after c-Myc reduction (Bottom). ( $J$ and $K$ ) Western blot showing ectopic expression of c-Myc in LNCaP and LAPC4 cells (Top) and transcript levels of KGA, GAC, and GLS1 after c-Myc overexpression (Bottom). (L) A model depicting how GLS1 isoform switch drives castration resistance and disease progression in PCa. PCA, prostate adenocarcinoma; Glu, glutamate; GIn, glutamine; $\mathrm{mCRPC}$, metastatic castration-resistant PCa; SCNC, small-cell neuroendocrine PCa. For each assay, $n=3$ replicates were performed. Data are expressed as mean \pm SD. $* P<0.05, * * P<0.01$, and $* * * P<0.001$ by two-tailed Student's $t$ test. n.s., not significant. 
understand the molecular mechanisms that up-regulate GAC expression after hormonal therapy and focused on c-Myc which mediates AR's ligand-independent function and drives disease progression toward CRPC (36). Relevant to our study, it has been reported that c-Myc up-regulates GAC expression (37-40). Bioinformatic analyses revealed that c-Myc was up-regulated in PCa, particularly in metastatic PCa (SI Appendix, Fig. S8A). IHC staining of TMA containing human PCa tissues and Western blot analysis of PCa cell lines showed that CRPC tissues and the castration-resistant CWRR1 and C4-2MDVR cells had the highest levels of c-Myc (SI Appendix, Fig. S8 B and C). In addition, in the longitudinal xenograft tumor model (Fig. $2 E$ ), c-Myc expression was elevated in the recurrent castration-resistant tumors in comparison to primary tumors (SI Appendix, Fig. S8D). These findings demonstrate strong association of c-Myc expression with castration resistance. Interestingly, we also discovered a strong positive correlation of c-Myc expression with GAC but not with KGA by studying the TCGA dataset (SI Appendix, Fig. S8E). Knocking down c-Myc in advanced PCa cells (LNCaP95 and C42MDVR) reduced the expression of GAC (Fig. $5 H$ and $I$ ). Conversely, overexpressing c-Myc in hormone-sensitive PCa cells (LNCaP and LAPC4) increased GAC levels (Fig. $5 J$ and $K$ ). In contrast, manipulation of c-Myc expression did not change KGA expression (Fig. $5 \mathrm{H}-\mathrm{K}$ ). These results suggest that c-Myc promotes the expression of the enzymatically potent GAC which may contribute to therapy resistance and disease progression.

Recurrent PCa after hormonal therapy usually maintains adenocarcinoma histology (CRPC-adeno), but a subset of recurrent tumors belongs to a variant histologic type known as SCNC which is composed of pure NE tumor cells with extremely aggressive behavior (41). Interestingly, MYC family members have been implicated in governing the histological differentiation of $\mathrm{PCa}$ in the advanced stages. c-Myc amplification was frequently detected in the CRPC tumors with adenocarcinoma histology but not in SCNC, both in human tissues and in an NE cell model (NCI-H660) (SI Appendix, Fig. S8 $B$ and $C$ ). In contrast, $N$-Myc was amplified and overexpressed in SCNC (SI Appendix, Fig. $\mathrm{S} 8 G)(42,43)$, while there was no significant up-regulation of c-Myc in SCNC (SI Appendix, Fig. S8F). Although $N$-Myc and c-Myc are expressed in advanced $\mathrm{PCa}$ of different histologies (CRPC-adeno vs. SCNC, respectively), $\mathrm{N}$-Myc similarly promoted the expression of GAC, not KGA, in LNCaP and C4-2 cells (SI Appendix, Fig. S8 $H$ and $I$ ).

AR inhibition promotes the emergence of SCNC through lineage plasticity $(44,45)$. We found that instead of inducing GAC expression, AR inhibition alone reduced GAC expression in LNCaP cells which does not express c-Myc or $N$-Myc, a finding consistent with the previous results (Fig. $5 C$ ). However, when $N$-Myc is overexpressed, ADT led to an eightfold increase of GAC (SI Appendix, Fig. S8I), suggesting that ADT and $N$-Myc cooperate to induce the expression of GAC, resulting in increased glutamine utilization and aggressive behavior of SCNC.

The above results firmly establish how AR, c-Myc, and $N$-Myc function to induce the isoform switch of GLS1 in PCa during the process of hormonal therapy, treatment resistance, and disease progression. However, the isoform switch from KGA to GAC could also be due to differences in RNA stabilities. To rule out this possibility, we treated LNCaP and PC3 cells, which express predominantly KGA and GAC, respectively, with actinomycin D (ActD) to inhibit GLS1 gene transcription. We observed no significant differences of RNA decay rates between $K G A$ and $G A C$ (SI Appendix, Fig. S8 $J$ and $K$ ), indicating that the isoform switch is not due to different mRNA stability.

\section{Discussion}

Despite decades of research, how AR activation promotes $\mathrm{PCa}$ cell proliferation remains largely unknown. Consequently, the molecular mechanisms of hormonal therapy and the subsequent development of therapy resistance are poorly understood. Our study has uncovered previously unrecognized mechanisms of action for AR and hormonal therapy in PCa: 1) Glutamine is an important nutrient for PCa, particularly in advanced, therapyresistant, and aggressive PCa. 2) AR binds to the AREs within the 3'UTR of the GLS1 gene, promotes GLS1 expression, and regulates transcription-coupled RNA splicing to favor the production of the KGA isoform. As a result, KGA is the predominant isoform of GLS1 in untreated, hormone-sensitive PCa, and $\mathrm{AR}$ is required for PCa cell survival. 3) Hormonal therapy suppresses AR function and GLS1 (predominantly the KGA isoform) expression, thus inhibiting glutamine catabolism and tumor cell survival. 4) Eventually, PCa cells regain the ability to utilize glutamine and resume proliferation, leading to therapy resistance and disease progression to the castration-resistant stage clinically. 5) The MYC family proteins c-Myc and $N$-Myc play important roles in this process along with AR. For many tumors at this stage, AR expression is maintained, and an important ligandindependent function of $\mathrm{AR}$ is transcriptional activation of c-Myc (36) which in turn induces GAC expression. Due to GAC's extremely potent enzymatic activity, the tumor cells can utilize glutamine more efficiently, resulting in aggressive behavior. This is characteristic of CRPC-adeno with expression of AR and c-Myc. 6) A significant number of tumors at this stage are histologically classified as SCNC in which GAC expression is equally important for the tumor cells' rapid proliferation and aggressive behavior. In SCNC, the increased expression of GAC is not mediated by c-Myc but by a combination of loss of AR expression (through lineage plasticity) $(45,46)$ and $N$-Myc overexpression (43). These important findings are depicted in Fig. $5 L$.

Our study has important clinical implications: 1) Currently used hormonal therapy targeting AR has significant side effects including sexual dysfunction and disorders of the cardiovascular and metabolic systems (47) because AR has diverse physiological functions in many organs and cells. A therapeutic strategy directly targeting GLS1 isoforms but not interfering with AR's physiological function in normal tissue can potentially have a therapeutic efficacy similar to or better than hormonal therapy but with much fewer side effects. 2) Due to the complexity of the intracellular signaling networks and their extensive cross-talks, PCa cells can quickly evolve to adapt to an androgen-deprived environment by a variety of mechanisms, such as activation of glucocorticoid receptor signaling (48), production of constitutively active AR splicing variants lacking the ligand-binding domain (1), and transdifferentiation toward an NE phenotype (45), resulting in tumor recurrence (CRPC-adeno or SCNC). A therapeutic strategy targeting GLS1 will bypass the complex upstream signaling pathways and directly suppress the production of energy and building blocks required by PCa cells, starving tumor cells to death. Since metabolic activity directly controls cellular proliferation $(49,50)$, it may be more difficult for the tumor cells to overcome a metabolic inhibition to develop resistance. 3) ARtargeted treatment approaches will not work for PCa cells in which AR activity is not required such as in AR indifferent tumors (30) and SCNC (12). Our study indicates that glutamine addiction is common across the entire spectrum of PCa including tumors lacking the expression of AR. In fact, advanced tumors and tumors independent of AR activity are more addicted to glutamine. As a result, many patients, particularly those who have exhausted currently available treatment options, will benefit from a treatment strategy targeting glutamine catabolism. CB-839, a specific small-molecular inhibitor of GLS1, has been evaluated in multiple clinical trials for solid and hematological malignancies as a single agent and in combination with an immune checkpoint inhibitor (38). Our data demonstrate that advanced and therapy-resistant $\mathrm{PCa}$, where GAC is overexpressed, are highly sensitive to CB-839 inhibition. GLS1 inhibitor may thus provide clinical benefits. 
There are still important yet unanswered questions related to the development of advanced PCa. An important factor in SCNC is hypoxia mediated by the master NE-differentiation regulator ONECUT2 (51). Future studies are needed to address if hypoxia affects the GLS1 isoforms and glutamine utilization in SCNC. Additionally, the molecular and cellular events described above are consistent with an "adaptation" model which states that PCa cells switch isoform expression from the AR-dependent KGA isoform to the androgen-independent GAC isoform in order to survive the androgen-deprived environment, resulting in more efficient glutamine utilization and more aggressive behavior seen in recurrent tumors (CRPC-adeno or SCNC). However, the findings may also be explained by the "clonal selection" model. This alternative model states that in untreated and hormone-sensitive $\mathrm{PCa}$, the tumor cells are heterogeneous with KGA being the predominant isoform in the majority of the tumor cells, while a minority of tumor cells express GAC predominantly. Hormonal therapy creates an androgen-deprived environment leading to the inhibition of KGA-expressing tumor cells and the selection of GAC-expressing tumor cells, and the latter eventually populate the recurrent tumors. Further studies are needed to distinguish these two interesting possibilities.

\section{Materials and Methods}

See SI Appendix for further information.

Cell Culture, Proliferation Assay, Colony Formation Assay, Gene Silence, and Overexpression. Cells were maintained under standard or specific conditions (for details, see SI Appendix). Cell viability assay and colony formation assay are described in SI Appendix. Cells used in each experiment were performed under identical and well controlled conditions to test a specific hypothesis. However, conditions such as the starting cell numbers and the sizes of the tissue culture plates were not identical among different experiments described in different figures. Therefore, the results of different experiments may not be directly comparable. shRNA lentiviral plasmids, Crispr single guide RNA (sgRNA) plasmids, specific siRNA pool, and ORF plasmids were either commercially purchased or kindly given from collaborators. Detailed protocols are described in SI Appendix.

1. E. S. Antonarakis et al., AR-V7 and resistance to enzalutamide and abiraterone in prostate cancer. N. Engl. J. Med. 371, 1028-1038 (2014).

2. C. E. Barbieri, M. A. Rubin, Molecular characterization of prostate cancer following androgen deprivation: The devil in the details. Eur. Urol. 66, 40-41 (2014).

3. T. Chandrasekar, J. C. Yang, A. C. Gao, C. P. Evans, Mechanisms of resistance in castration-resistant prostate cancer (CRPC). Transl. Androl. Urol. 4, 365-380 (2015).

4. P. A. Watson, V. K. Arora, C. L. Sawyers, Emerging mechanisms of resistance to androgen receptor inhibitors in prostate cancer. Nat. Rev. Cancer 15, 701-711 (2015).

5. C. Tran et al., Development of a second-generation antiandrogen for treatment of advanced prostate cancer. Science 324, 787-790 (2009).

6. M. Reina-Campos et al., Increased serine and one-carbon pathway metabolism by PKC $/ 1$ deficiency promotes neuroendocrine prostate cancer. Cancer Cell 35, 385-400.e9 (2019).

7. M. Nadal et al., Structure of the homodimeric androgen receptor ligand-binding domain. Nat. Commun. 8, 14388 (2017)

8. D. A. Quigley et al., Genomic hallmarks and structural variation in metastatic prostate cancer. Cell 174, 758-769.e9 (2018)

9. F. Vazquez et al., PGC1 $\alpha$ expression defines a subset of human melanoma tumors with increased mitochondrial capacity and resistance to oxidative stress. Cancer Cell 23, 287-301 (2013).

10. A. Viale et al., Oncogene ablation-resistant pancreatic cancer cells depend on mitochondrial function. Nature 514, 628-632 (2014).

11. G. Zhang et al., Targeting mitochondrial biogenesis to overcome drug resistance to MAPK inhibitors. J. Clin. Invest. 126, 1834-1856 (2016).

12. H. Beltran et al., Divergent clonal evolution of castration-resistant neuroendocrine prostate cancer. Nat. Med. 22, 298-305 (2016).

13. J. W. Park, J. K. Lee, O. N. Witte, J. Huang, FOXA2 is a sensitive and specific marker for small cell neuroendocrine carcinoma of the prostate. Mod. Pathol. 30, 1262-1272 (2017).

14. S. Tai et al., PC3 is a cell line characteristic of prostatic small cell carcinoma. Prostate 71, 1668-1679 (2011).

15. W. Li et al., The role of CD44 in glucose metabolism in prostatic small cell neuroendocrine carcinoma. Mol. Cancer Res. 14, 344-353 (2016).

16. L. Xu et al., ATM deficiency promotes progression of CRPC by enhancing Warburg effect. Endocr. Relat. Cancer 26, 59-71 (2019).

17. E. Eidelman, J. Twum-Ampofo, J. Ansari, M. M. Siddiqui, The metabolic phenotype of prostate cancer. Front. Oncol. 7, 131 (2017).
Tissue Microarrays and Immunohistochemistry. Tissue microarrays, including treatment-naive, adjacent benign, CRPC and SCNC samples, were constructed and have been reported previously $(13,52)$. Immunohistochemistry was performed following standard procedures, and the Quick-score system was applied to determine the intensity of staining (13).

Metabolite Extraction, Isotope Tracing, and Mass Spectrometry. As described previously (53), cells were harvested from 6-well plate after corresponding treatment. For isotope-tracing analysis, medium was replaced by fresh glutamine/glucose-deprived medium supplemented with $2 \mathrm{mM}{ }^{13} \mathrm{C}$-glutamine or $4.5 \mathrm{~g} / \mathrm{L}{ }^{13} \mathrm{C}$-glucose isotope. Metabolites were extracted in $80 \%$ cold methanol followed by speed vacuum drying. The dried pellets were sent for further LC/MS analyses.

Minigene Assay. The gBlock, which contains exon 14-19 and their flanking intron regions of GLS1 gene, was synthesized by Integrated DNA Technologies and used as the template for PCR amplification. The DNA fragment product was cloned into the plasmid vector pRL-TK (Promega) between Nhel and $\mathrm{Xbal}$ sites. DNA sequencing confirmed the integrity of the final construct, which was subsequently transfected into desired cells. KGA- and GACspecific primers were used to determine the transcript levels by qPCR.

Animal Experiments. For drug efficacy experiments, LNCaP and PC3 cells were injected subcutaneously in mice. For each tumor type, mice were randomly distributed into two groups receiving either vehicle or CB-839 $(200 \mathrm{mg} / \mathrm{kg}$ oral administration) (32). For longitudinal observation of the isoform switch, surgical castration was performed when subcutaneously implemented tumors reached $\sim 200 \mathrm{~mm}^{3}$. Animals were killed after tumor recurred significantly or tumor volumes exceeded $1,000 \mathrm{~mm}^{3}$. Harvested tumors were embedded into paraffin followed by hematoxylin and eosin staining and immunohistochemistry staining.

Data availability. All study data are included in the article and/or supporting information.

ACKNOWLEDGMENTS. We thank Drs. Yen-Nien Liu, Qing Cheng, Fuwen Yuan, and Anmbreen Jamroze for their valuable discussion and comments. This work was supported by the Department of Defense (DOD-W81XWH-19 1-0411 to J.H.), the Prostate Cancer Foundation Movember Valor Challenge Award (2018), and the National Cancer Institute (K99-K99CA237618 to X.G.).

18. E. Dardenne et al, $\mathrm{N}$-myc induces an EZH2-mediated transcriptional program driving neuroendocrine prostate cancer. Cancer Cell 30, 563-577 (2016).

19. Y. Yin et al., N-Myc promotes therapeutic resistance development of neuroendocrine prostate cancer by differentially regulating miR-421/ATM pathway. Mol. Cancer 18, 11 (2019).

20. J. K. Lee et al, $N$-myc drives neuroendocrine prostate cancer initiated from human prostate epithelial cells. Cancer Cell 29, 536-547 (2016).

21. B. J. Altman, Z. E. Stine, C. V. Dang, From Krebs to clinic: Glutamine metabolism to cancer therapy. Nat. Rev. Cancer 16, 619-634 (2016)

22. A. A. Cluntun, M. J. Lukey, R. A. Cerione, J. W. Locasale, Glutamine metabolism in cancer: Understanding the heterogeneity. Trends Cancer 3, 169-180 (2017).

23. C. T. Hensley, A. T. Wasti, R. J. DeBerardinis, Glutamine and cancer: Cell biology, physiology, and clinical opportunities. J. Clin. Invest. 123, 3678-3684 (2013).

24. S. Suzuki et al., Phosphate-activated glutaminase (GLS2), a p53-inducible regulator of glutamine metabolism and reactive oxygen species. Proc. Natl. Acad. Sci. U.S.A. 107, 7461-7466 (2010)

25. L. Xiang et al., Knock-down of glutaminase 2 expression decreases glutathione, $\mathrm{NADH}$, and sensitizes cervical cancer to ionizing radiation. Biochim. Biophys. Acta 1833, 2996-3005 (2013)

26. C. V. Dang, MYC, microRNAs and glutamine addiction in cancers. Cell Cycle 8 , 3243-3245 (2009)

27. A. Cassago et al., Mitochondrial localization and structure-based phosphate activation mechanism of Glutaminase $\mathrm{C}$ with implications for cancer metabolism. Proc. Natl. Acad. Sci. U.S.A. 109, 1092-1097 (2012).

28. Y. Li et al., Targeting cellular heterogeneity with CXCR2 blockade for the treatment of therapy-resistant prostate cancer. Sci. Transl. Med. 11, eaax0428 (2019).

29. C. P. Masamha et al., CFIm25 regulates glutaminase alternative terminal exon definition to modulate miR-23 function. RNA 22, 830-838 (2016)

30. Q. Li et al., Linking prostate cancer cell AR heterogeneity to distinct castration and enzalutamide responses. Nat. Commun. 9, 3600 (2018)

31. R. Romero et al., Keap1 loss promotes Kras-driven lung cancer and results in dependence on glutaminolysis. Nat. Med. 23, 1362-1368 (2017)

32. M. I. Gross et al., Antitumor activity of the glutaminase inhibitor CB-839 in triplenegative breast cancer. Mol. Cancer Ther. 13, 890-901 (2014).

33. S. J. Barfeld, H. M. Itkonen, A. Urbanucci, I. G. Mills, Androgen-regulated metabolism and biosynthesis in prostate cancer. Endocr. Relat. Cancer 21, T57-T66 (2014). 
34. Z. Chen et al., Agonist and antagonist switch DNA motifs recognized by human androgen receptor in prostate cancer. EMBO J. 34, 502-516 (2015).

35. Q. Wang et al., Androgen receptor regulates a distinct transcription program in androgen-independent prostate cancer. Cell 138, 245-256 (2009).

36. L. Gao et al., Androgen receptor promotes ligand-independent prostate cancer progression through c-Myc upregulation. PLoS One 8, e63563 (2013).

37. P. Gao et al., c-Myc suppression of miR-23a/b enhances mitochondrial glutaminase expression and glutamine metabolism. Nature 458, 762-765 (2009).

38. M. Lampa et al., Glutaminase is essential for the growth of triple-negative breast cancer cells with a deregulated glutamine metabolism pathway and its suppression synergizes with mTOR inhibition. PLoS One 12, e0185092 (2017)

39. D. R. Wise et al., Myc regulates a transcriptional program that stimulates mitochondrial glutaminolysis and leads to glutamine addiction. Proc. Natl. Acad. Sci. U.S.A. 105 18782-18787 (2008)

40. M. Yuneva, N. Zamboni, P. Oefner, R. Sachidanandam, Y. Lazebnik, Deficiency in glutamine but not glucose induces MYC-dependent apoptosis in human cells. J. Cell Biol. 178, 93-105 (2007).

41. R. Aggarwal et al., Clinical and genomic characterization of treatment-emergent small-cell neuroendocrine prostate cancer: A multi-institutional prospective study. J. Clin. Oncol. 36, 2492-2503 (2018).

42. D. S. Rickman, J. H. Schulte, M. Eilers, The expanding world of $N$-MYC-driven tumors. Cancer Discov. 8, 150-163 (2018).
43. H. Beltran et al., Molecular characterization of neuroendocrine prostate cancer and identification of new drug targets. Cancer Discov. 1, 487-495 (2011).

44. S. Kregel et al., Sox 2 is an androgen receptor-repressed gene that promotes castration-resistant prostate cancer. PLoS One 8, e53701 (2013).

45. P. Mu et al., SOX2 promotes lineage plasticity and antiandrogen resistance in TP53and RB1-deficient prostate cancer. Science 355, 84-88 (2017).

46. S. Y. Ku et al., Rb1 and Trp53 cooperate to suppress prostate cancer lineage plasticity, metastasis, and antiandrogen resistance. Science 355, 78-83 (2017).

47. S. C. Manolagas, C. A. O'Brien, M. Almeida, The role of estrogen and androgen receptors in bone health and disease. Nat. Rev. Endocrinol. 9, 699-712 (2013).

48. V. K. Arora et al., Glucocorticoid receptor confers resistance to antiandrogens by bypassing androgen receptor blockade. Cell 155, 1309-1322 (2013).

49. M. A. White, D. E. Frigo, Regulation of SLC1A4 and SLC1A5 in prostate cancerresponse. Mol. Cancer Res. 16, 1811-1812 (2018).

50. N. M. Zacharias et al., Metabolic differences in glutamine utilization lead to metabolic vulnerabilities in prostate cancer. Sci. Rep. 7, 16159 (2017).

51. H. Guo et al., ONECUT2 is a driver of neuroendocrine prostate cancer. Nat. Commun. 10, 278 (2019).

52. J. Huang et al., Differential expression of interleukin-8 and its receptors in the neuroendocrine and non-neuroendocrine compartments of prostate cancer. Am. J. Pathol. 166, 1807-1815 (2005).

53. M. Pan et al., Regional glutamine deficiency in tumours promotes dedifferentiation through inhibition of histone demethylation. Nat. Cell Biol. 18, 1090-1101 (2016). 